\title{
Geodetic Contraction Games on Graphs
}

\author{
By A. S. Fraenkel ${ }^{1}$ and F. Harary ${ }^{2}$
}

\begin{abstract}
Let $G=(V, E)$ be a finite graph. The set $L$ of labeled vertices is initially empty. Two players A and B move alternately (A first), by choosing an unlabled vertex $u \in V \backslash L$; then $u$ itself and all vertices on shortest paths between $u$ and any vertex of $L$ are adjoined to $L$. When $L=V$, the game is over. In normal play, the first player unable to move loses and his opponent wins. The outcome is reversed for misère play. We resolve the game by determining its winning strategies for the following cases: trees in normal play; cycles in normal and misère play; and complete graphs $K_{m}$ with rays all of length $n$, in normal play.
\end{abstract}

\section{Introduction}

Our purpose is to investigate geodetic contraction games, or geodetic games for short, as defined in the abstract, on a number of graphs.

These games, whether played in normal or misère form, are impartial, in the sense that from any position exactly the same moves are legal for both players. We follow Berlekamp, Conway and Guy [1] in defining a P-position as any position $u$ from which the Previous player (B) can force a win, that is, the player who responds to the move made from $u$. An $N$-position is any position $v$ from which the $N$ ext player (A) can force a win, that is, the player who moves from $v$. The set of all $P$-positions is denoted by $\mathscr{P}$ and the set of all $N$-positions by $\mathscr{N}$. In general, knowing that a position is in $\mathscr{P}$ in normal play does not tell us whether it is in $\mathscr{P}$ or in $\mathscr{N}$ in misère play.

In Section 2 we present a polynomial strategy for geodetic games on trees in normal play. It leans heavily on Úlehla's strategy for Hackendot [3] (see also "Bushenhack" in [1]). In Section 3 we determine the $P$ and $N$ positions for a cycle $C_{n}$ with $n$ vertices for normal play, and in Section 4 we do the same for misère play. Let $K_{m}$ - $R_{n}$ denote the complete graph on $m$ vertices with a ray of length $n$ impinging on each of its $m$ vertices. In the final Section 5 we prove the somewhat surprising result that in normal play $K_{m} \circ R_{n} \in \mathscr{P}$ for all $m \geq 2$ and $n \geq 2$.

1 Department of Applied Mathematics and Computer Science, The Weizmann Institute of Science, Rehovot 76100, Israel. Partial support by Canadian Research Grants 69-0695 and A-4792 during visits at the University of Calgary and Simon Fraser University is gratefully acknowledged.

2 The University of Michigan, Ann Arbor, MI 48109. Present address: Department of Computer Science, New Mexico State University, Las Cruces, NM 88003. 


\section{Trees}

To analyze a geodetic game on a tree in normal play, we resort to von Neumann's Hackendot, played on a rooted tree $T$, directed away from the root $r$. Player A selects any vertex $a$. There is a unique (directed) path from $r$ to $a$. All vertices on this path are deleted, leaving a forest $T^{a}$. If $T^{a}$ is nonempty, B selects a vertex $b$ in it and deletes all vertices on the path joining it to a root. Provided that the resulting forest $T^{a b}$ is nonempty, A selects a further vertex $c$ and forms $T^{a b c}$, etc. Úlehla [3] gave a polynomial strategy for this game in normal play. Using this fact we easily obtain the first observation.

Theorem 1 : Let $T$ be an undirected finite tree on which a geodetic game is played. Let $u$ be the node labeled in the first move. Let $T^{\prime}$ be a directed tree derived from $T$, namely the tree $T$ with root $u$, oriented away from $u$, that is, for any node $v \neq$ $u$, direct the path $(u, v)$ from $u$ to $v$. Then normal play of the geodetic game on $T$ after the first node $u$ has been labeled, denoted by $T^{u}$, is equivalent to the Hackendot game $T^{\prime} u$ (the Hackendot game on $T^{\prime}$, after the first move of removing the root $u)$.

Proof: If $\Gamma_{1}$ and $\Gamma_{2}$ are two games, their sum $\Gamma=\left(\Gamma_{1}, \Gamma_{2}\right)$ is the game played as follows: A player at his turn selects a move from either $\Gamma_{1}$ or $\Gamma_{2}$. To show that $\Gamma_{1}$ and $\Gamma_{2}$ are equivalent, that is, that they have the same outcome (in fact, the same Sprague-Grundy function-values), it suffices to show that $\left(\Gamma_{1}, \Gamma_{2}\right) \in \mathscr{P}$ (see [1]). For any move $T^{u v}$ by $\mathrm{A}$, the labeled vertices are the same as the removed ones in $T^{\prime} u v$ and conversely. Therefore B can make a last move, hence $\left(T^{u}, T^{\prime} u\right) \in \mathscr{P}$.

Corollary 1: A geodetic game on a finite undirected tree $T$ in normal play has a polynomial decision procedure.

Proof: The procedure is to label some node $u_{1}$ of the tree $T$ and orient the tree away from $u_{1}$, resulting in a directed tree $T^{\prime}$. If the first player can win Hackendot by picking $u_{1}$ as the first move in $T^{\prime}$, then the first player can also win in the geodetic game. Otherwise we try the same procedure with the first player picking some $u_{2}$ $\neq u_{1}$ in $T$, and so on. If the first player loses in Hackendot whenever he picks the root for all possible $T^{\prime}$ induced by $T$, then the second player can win in the geodetic game.

Questions: (i) Is there a more efficient strategy? (ii) Do geodetic games on forests have a polynomial strategy? On directed acyclic graphs with all roots initially labeled?

Corollary 2: If $T$ with at least two vertices is an undirected finite tree on which a geodetic game is played, a first winning move cannot be to label a vertex $u$ with degree $d(u)=1$. 
Proof: The corresponding move in $T^{\prime}$ leaves another directed tree which is a Hackendot $N$-position, since every directed tree is.

\section{Cycles - Normal Play}

Let $C_{n}$ be the cycle of length $n$, and write $V\left(C_{n}\right)=\{0,1, \ldots, n-1\}$. Without loss of generality, A always chooses vertex 0 on her first move. In this section we consider normal play. When $n$ is even, the game is quite trivial as B selects $n / 2$ and he wins at once since now $L=V$. So $n$ is odd in the rest of this section.

After A chooses point 0 , the result can be regarded as a rooted cycle, denoted by $C_{n}^{*}$. Before stating and proving the theorem solving the game for normal play on a cycle, we illustrate for small odd $n$.

In [2], a shrewd move is (jokingly) defined as a move which always results in a unique configuration, up to isomorphism, and hence cannot affect the outcome of the game. When $n=3$, all moves are shrewd! After A picks 0 forming $C_{3}^{*}$, B chooses either of the two remaining vertices so $A$ moves last and wins.

When $n=5$, after A has moved to $C_{5}^{*}$, B has two possibilities: he can pick either of the vertices 1 or 2 . If he picks 1 , he effectively forms $C_{4}^{*}$ and loses. So he picks vertex 2 forming $C_{3}^{*}$ and wins as we just saw.

Figure 1 illustrates $n=7$. After A makes $C_{7}^{*}$ on her first move, Figure 1(a), B can form any one of $C_{6}^{*}, C_{5}^{*}$ or $C_{4}^{*}$ on his first move by picking point 1,2 , or 3 as in Figure 1 $(b, c$ or d). The only one of these which does not lose immediately for $B$ is $C_{5}^{*}$. But then A moves $C_{5}^{*} \rightarrow C_{3}^{*}$ and wins, as we saw above. So $\mathrm{A}$ wins $C_{7}$.

Theorem 2: For normal play, $C_{n} \in \mathscr{N}$ if and only if $n=2^{k}-1$ for some $k \geq 1$.

$C_{7}^{*}$

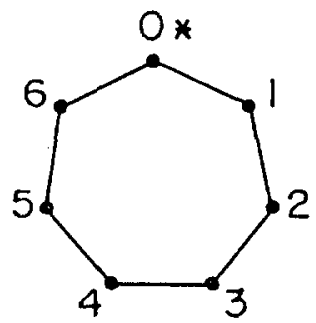

(a)
$C_{6}^{*}$

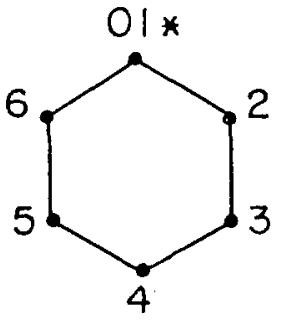

(b)
$C_{5}^{*}$

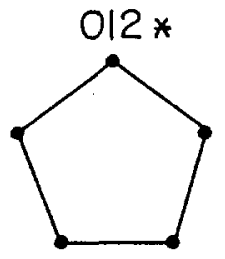

(c)
$C_{4}^{x}$

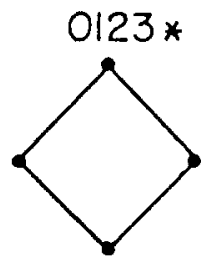

(d)

Fig. 1. A geodetic game on the 7-cycle (normal play) 
Proof: We have already seen how this game goes for small $n$. For arbitrary odd $n$ $=2 m+1$, after $\mathrm{A}$ moves to $C_{n}^{*}, \mathrm{~B}$ can form any one of $C_{n-1}^{*}, C_{n-2}^{*}, \ldots, C_{\mathrm{n}-m}^{*}$. There are two possibilities. If $n$ is not of the form $2^{k}-1$, then exactly one of the numbers in the closed integral interval $[n-m, n-1]=\{n-1, n-2, \ldots, n-m\}$ is one of that form and $\mathrm{B}$ moves to that one. If $n=2^{k}-1$, then no number in that interval has this form. Thus no matter what first move B makes, A can get the smaller rooted cycle of length $2^{k-1}-1$ on her second move, and reduce it eventually to the small cycles above, thus winning the game.

\section{Cycles - Misère Play}

This game is even easier to analyze and was first settled by Frederick Teague, a student of the second author.

Theorem 3: For misère play, $C_{n} \in \mathscr{N}$ if and only if $n=2^{k}$ for some $k \geq 1$.

Proof: Again using induction, when $n=2$ or 3 , all moves are shrewd and A wins the former and loses the latter. When $n=4$, after A makes $C_{4}^{*}, \mathrm{~B}$ loses on the very next move unless he makes $C_{3}^{*}$ which also loses for B so A wins. For arbitrary $n=$ $2^{k}$, A shrewdly moves to $C_{n}^{*}$ and after any first move by $\mathrm{B}$, the second move by $\mathrm{A}$ forms $C_{2^{k-1}}^{*}$, and so forth, resulting in victory by $\mathrm{A}$.

When $n \neq 2^{k}$, A must still open with $C_{n}^{*}$ but now B can always move to rooted cycles whose length is a power of 2 , so $B$ wins.

\section{Complete Graphs With Rays}

In this section we investigate the geodetic game on $K_{m} \circ R_{n}$ in normal play, where $K_{m}$ is the complete graph on $m$ vertices and $R_{n}$ is a ray of $n$ vertices (a ray of length $n$ ), impinging on each of the $m$ vertices of $K_{m}$. The vertices of the rays are numbered from 1 on $K_{m}$ to $n$ at the far end of the ray. We will identify vertices on any given ray with their serial numbers. A few graphs of the form $K_{m} \circ R_{n}$ are displayed in Figure 2. 


\section{$\overbrace{0}^{1}$ \\ $K_{3}=K_{3} \circ R_{1}$}

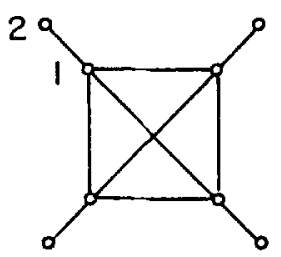

$K_{4} \circ R_{2}$

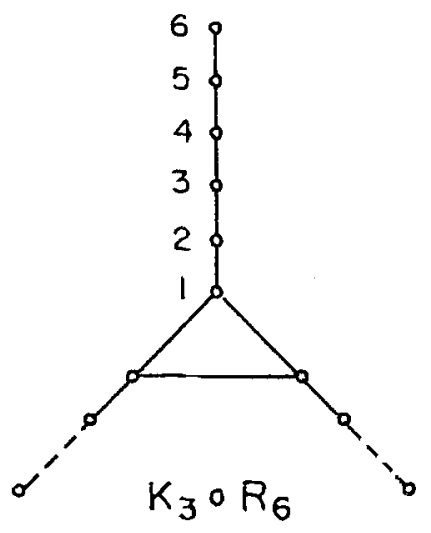

(c)

\section{)}

Fig. 2. Several instances of $K_{m} \circ R_{n}$

The main result of this section can now be stated.

Theorem 4: For normal play, $K_{m} \circ R_{n} \in \mathscr{P}$ for all $m \geq 2$ and $n \geq 2$.

Proof: The assertion is clearly true when $m$ is even, since then the graph has central symmetry - but without a central vertex! - and so B can play symmetrically with respect to the center (see e.g. Figure $2 b$ ). So henceforth $m$ will be odd.

We shall distinguish between two phases of play: Phase I, where all previous moves, if any, were made on a single ray only, and not all of it has been labeled yet; and Phase II, where vertices on more than one ray have been labeled, or one ray has been labeled entirely.

In Phase II the graph is easily shown to be equivalent to a star whose center is the collection of labeled vertices, coalesced into a single labeled vertex. Any move in this phase in effect shortens precisely one of the rays by any desired amount. In other words, we have an $m$-pile or $(m-1)$-pile Nim game, and the player making the Nim-sum 0 is the winner (see e.g. [1]). A star with Nim-sum 0 will be called 0 -star. Otherwise the star is a nonzero star. If, for example, A labeled 4 in $K_{5} \circ R_{5}$ (Figure $3 a)$, which is a move in Phase I, B can pass to Phase II by labeling 1 on another ray (denoted 1'), which produces the 0 -star of Figure $3 \mathrm{~b}$. (The three dotted edges in Figure $3 \mathrm{~b}$ do not influence the outcome of the game.) 


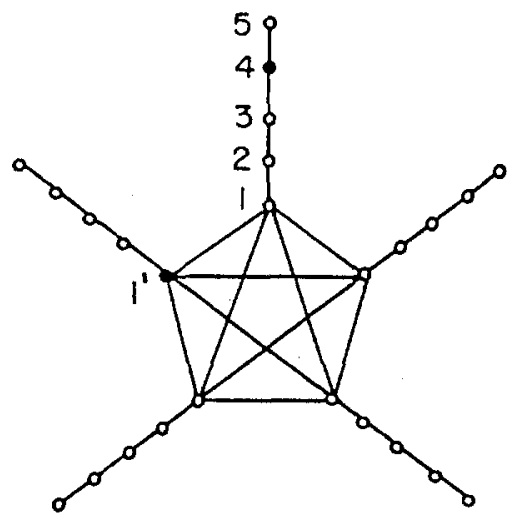

(a)

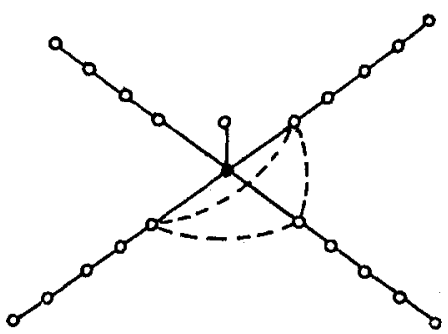

(b)

Fig. 3. Passing from Phase I to Phase II

We may assume that a player will pass from Phase I to Phase II if and only if he can do so by means of a move which will transform the graph into a 0-star. Because if he passes to Phase II with a nonzero star, then his opponent can make a 0 -star and win. Note that also when a player has no choice but to pass from Phase I to Phase II, he can do so by making the graph a 0-star.

Note that in Phase I a player who first labels one of the end vertices, 1 or $n$, is in a losing position, as his opponent can pass to Phase II with a 0 -star by labeling the other end vertex. tions.

To complete the proof of Theorem 4, we use three definitions and eight proposi-

\section{Definitions}

1. An extreme vertex is the largest labeled vertex on a ray. Thus if vertex $n-i$ has been labeled on a ray but $n-i+1, \ldots, n$ are unlabeled, then vertex $n-i$ is extreme.

A labeled vertex which is not extreme is called nonextreme.

2. An effective ray is the unlabeled far end part of a ray, that is, the ray consisting of the end vertices not yet labeled. Thus after the first move of $\mathrm{A}$ in the example of Figure 3, we have one effective ray of length 1 and four effective rays of length 5. After the response of $B$ we have one effective ray of length 1 , one effective ray of length 4 , one effective ray of length 5 (which together already form a 0 -star) and two additional effective rays of length 5 (which preserve the 0 -star). 
3. Let $\mathscr{C}$ be the class of moves in Phase I such that the next move cannot produce a 0 -star. Note that if $M$ is a move in $\mathscr{C}$, then both 1 and $n$ are still unlabeled after the move $M$. For example, a first move of A of labeling 5 on $K_{3} \circ R_{6}(\mathrm{Fi}-$ gure $2 \mathrm{c}$ ) is in $\mathscr{E}$, since there is no next move yielding a 0 -star.

Remark: A good response to A's move in the last example is to label vertex 2, which preserves the effective ray of length 1 , and is thus a move in $\mathscr{E}$. Note, however, that labeling 2 as a first move is not a move in $\mathscr{E}$, since it can be countered by transforming one of the as yet unlabeled rays into an effective ray of length 2 , thus producing a 0 -star. This shows that $\mathscr{C}$ is move-dependent.

It should be clear that player A's only chance to win is to make moves in $\mathscr{C}$. We shall show, however, that for every move of $\mathrm{A}$ in $\mathscr{C}, \mathrm{B}$ can respond with another move in $\mathscr{C}$, so $\mathrm{A}$ will eventually have to make a move in Phase I such that the next move - to be made by B! - can transform the graph into a 0 -star.

We denote by $a \oplus b$ the Nim-sum of $a$ and $b$.

Proposition 1: A move $M$ in Phase I leaving an effective ray of length $\ell$ is in $\mathscr{C}$ if and only if $n \oplus \ell>n$ except for the case $n=2^{s}(s \geq 1)$ and $\ell=n-1$, for which $n \oplus$ $\ell>n$ but the move is not in $\mathscr{E}$.

Proof: Suppose first $n \oplus \ell>n$.

At the end of move $M$ we have three effective rays of lengths $n, n$ and $\ell$. In addition there is an even number of effective rays of lengths $n$ (whose Nim-sum is 0 ). How can a single move transform this position into a 0 -star?

Case I: Transform the effective ray of length $\ell$ into one of length 0 . This can be done only if the move $M$ consisted of labeling 1, that is, $\ell=n-1$. Then $n \oplus \ell>n$ if and only if $n=2^{s}(s \geq 1)$. This move $M$ is not in $\mathscr{C}$, since the next move of labeling $n$ results in a 0 -star.

Case II: Transform an effective ray of length $n$ into one of length $i<n$ such that $n \oplus \ell \oplus i=0$. But then $i=n \oplus \ell>n$, a contradiction. Thus $M \in \mathscr{C}$.

Now suppose $n \oplus \ell \leq n$.

If $n \oplus \ell=n$, then $M$ consisted of labeling $n$, so 1 can be labeled in reply, resulting in a 0 -star. If $n \oplus \ell<n$, then transforming one of the as yet unlabeled rays into an effective ray of length $n \oplus \ell$ results in a 0 -star.

Let lg denote $\log$ to the base 2 . As usual, $[a, b],(a, b]$ and $[a, b)$ denotes a closed interval, an interval open on the left and closed on the right, and an interval closed on the left and open on the right, respectively. We also denote by $n_{b}$ the binary encoding of $n$. The trivial proof of the next statement is omitted.

Proposition 2: Suppose $n_{b}$ - with least significant bit in position 0 and leading 1-bit in position $[\lg n]-$ has 0-bits precisely in the positions $j_{1}, \ldots, j_{k}$ satisfying

$0 \leq j_{1}<j_{2}<\ldots<j_{k}<[\lg n]$. 
Then an effective ray of length $\ell$ satisfies $\ell \oplus n>n$ if and only if $\ell \in$ $\left[2^{j_{i}}, 2^{j_{i}+1}\right)$, that is, if and only if the extreme vertex is in $\left(n-2^{j_{i}+1}, n-2^{j_{i}}\right]$ for any $i \in[1, k]$.

Here now is the winning strategy of player $B$.

Winning Strategy $W$ of $\mathrm{B}$ (moves restricted to single ray).

For any fixed $i \in[1, k]$, if A labels some unlabeled vertex $a_{i}=a\left(t_{i}\right)=n-2^{j_{i}}$ $-t_{i}$, then $\mathrm{B}$ responds by labeling $b_{i}=b\left(t_{i}\right)=2^{j_{1}}+\ldots+2^{j_{i-1}}+2+t_{i}$ for any $t_{i} \in$ $\left[0,2^{j_{t}}\right)$. Conversely, if after the first move A labels $b_{i}=2^{j_{1}}+\ldots+2^{j_{i-1}}+2+t_{i}$, then $\mathrm{B}$ responds by labeling $a_{i}=n-2^{j_{i}}-t_{i}$ for every $t_{i} \in\left[0,2^{\dddot{j}_{i}}\right)$.

The validity of $W$ as a winning strategy for B will be established by means of the next few propositions, which show: (i) B's response is always possible; (ii) it is in $\mathscr{C}$ whenever A's move is in $\mathscr{E}$; (iii) A has no moves in $\mathscr{C}$ other than those specified by $W$. By Propositions 1 and 2, the moves of $\mathrm{A}$ as given in $W$ are in $\mathscr{C}$ except when $n=2^{s}(s \geq 1)$ and A labels vertex $n$ or 1 as an extreme vertex. (In these two exceptional cases $W$ calls on $B$ to label 1 or $n$, which are winning moves, since they transform the graph into a 0-star.)

It may be helpful to follow the arguments below by means of an example, such as $K_{3} \circ R_{21}$ shown in Figure 4, for which $21_{b}=10101$, so $k=2, j_{1}=1, j_{2}=3$. The serial numbers of the vertices are to the left of the vertical ray. The numbering to the right of the ray indicates the potential moves specified by $W$ : An odd number $2 r-1$ indicates a move of $A$ which is in $\mathscr{C}$, and its successor $2 r$ indicates B's response which is also in $\mathscr{C}(1 \leq r \leq 10)$. For example, if A labels 5 , then $\mathrm{B}$ labels 6 ; if $\mathrm{A}$ labels 3 , then B labels 4 , etc.

Proposition 3: The vertex $b_{i}$ or $a_{i}$ which $W$ prescribes as B's response to A's move, is always different from $a_{i}$ or $b_{i}$ labeled by $\mathrm{A}$ in the preceding move.

Proof: Suppose $a_{i}=b_{i}$. Then

$$
n=2^{j_{1}}+\ldots+2^{j_{i}}+2+2 t_{i} \text {. }
$$

If $n$ is even then $j_{1}=0$, so the right-hand side is odd, a contradiction. If $n$ is odd then $j_{1}>0$, so the right-hand side is even, again a contradiction.

Let $\bar{n}$ denote the 2 's complement of $n$. Note that $\bar{n}_{b}$ is gotten from $n_{b}$ by interchanging all 1-bits of $n_{b}$ with 0 -bits and conversely, and adding a least significant 1-bit. Thus $\overline{21}_{b}=1011$. We have the following result. 


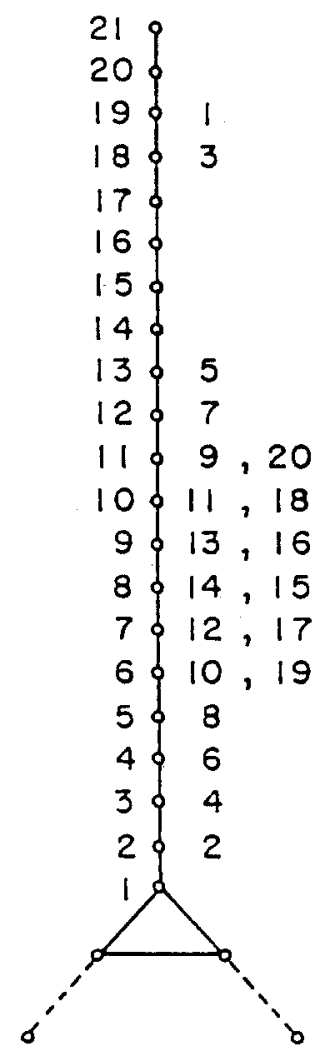

Fig. 4. $K_{3} \circ R_{21}$

Proposition 4: (i) $n+\bar{n}=2^{[\lg n]+1}$; (ii) $n-\bar{n}=2\left(n-2^{[\lg n])}\right.$; (iii) $\bar{n} \leq n$ (with equality if and only if $\left.n=2^{s}(s \geq 0)\right)$.

Proof: (i) is the definition of the 2's complement of $n$. Adding $n$ to both sides gives (ii). By (i),

$$
\bar{n}=2^{[\lg n]+1}-n \leq 2 n-n=n,
$$

since the most significant bit of $n_{b}$ is in position $[\lg n]$.

Proposition 5: (i) For $n \neq 2^{s}$ ( $s \geq 1$ ), the smallest extreme vertex in class $\mathscr{C}$ is $n-$ $2^{j k+1}+1$. (ii) The response of $\mathrm{B}$ specified by $W$ to this move of $\mathrm{A}$ is to label $\bar{n}$, which is the largest vertex of the form $b_{i}$ labeled by B. (iii) There exists $i$ such that $a_{i}<b_{i}$ if and only if $[\lg n]=j_{k}+1$, that is, if and only if $n_{b}$ has a leading 10 . (iv) The set of all moves of the form $b_{i}$ is the interval $[2, \bar{n}]$. (v) The set of all moves 
of the form $a_{i}$ is a collection of intervals, the longest of which is $I_{L}=\left(n-2^{j_{k}+1}\right.$, $\left.n-2^{j k-q}\right]$, where the run of the leftmost 0 -bits of $n_{b}$ occupies positions $j_{k}, j_{k}-$ $1, \ldots, j_{k}-q$ (but position $j_{k}-q-1$ contains a 1-bit for some $q \in\left[0, j_{k}\right.$ ) if $n \neq 2^{s}$ $(s \geq 1)$; or $q=j_{k}$ if $n=2^{s}(s \geq 1)$ ). (vi) The largest $a_{i}$ satisfying $a_{i}<b_{i}$, if it exists, is $a_{i}=2^{j_{k}} \in I_{L}$.

Proof: The smallest extreme vertex labeled by A as given by $W$ is $a=n-2^{j k}-$ $\left(2^{j_{k}}-1\right)=n-2^{j_{k}+1}+1$. This move is in $\mathscr{C}$ by Proposition 1 except when $n=$ $2^{S}(s \geq 1)$. The response specified by $W$ is to label

$$
b=2^{j_{1}}+\ldots+2^{j_{k-1}}+2+\left(2^{j_{k}}-1\right)=\bar{n},
$$

which is the largest vertex labeled by $B$ in response to an extreme vertex of the form $a_{i}$ labeled by A. This proves (i) and (ii).

(iii) If $[\lg n] \geq j_{k}+2$, that is, $n_{b}$ has a leading 11 , then $n>2^{j_{k}+2}$. Now $a_{i}<$ $b_{i}$ implies $n-2^{j_{k}+1}+1<\bar{n}$ by (i) and (ii), so $n<\Sigma_{i=1}^{k} 2^{j_{i}}+2^{j_{k}+1} \leq 2^{j_{k}+2}-1$, a contradiction. If $n_{b}$ has a leading 10 , then $n \leq 2^{j_{k}+1}+2^{j_{k}}-1$. Also $\bar{n} \geqslant 2^{j_{k}}+1$. Hence $n-2^{j_{k}+1}+1 \leq 2^{j_{k}}<\bar{n}$.

(iv) Since $b_{i}$ with $t_{i}=2^{j_{i}}-1$ is $b_{i+1}-1$ with $t_{i+1}=0$, we see that the intervals of all moves of the form $b_{i}$ and $b_{i+1}$ are directly adjacent. The upper bound $\bar{n}$ of the interval was established in (ii).

(v) The largest vertex in $I_{L}$ is $n-2^{j_{k}-q}$ by the definition of $q$, and the smallest is $n-2^{j_{k+1}}+1$ by (i).

(vi) The largest $a_{i}$ less than $b_{i}$ satisfies $a_{i}=b_{i}-1$ if $a_{i}, b_{i} \in I_{L}$. Then

$n-2^{j_{i}}-t_{i}=2^{j_{1}}+\ldots+2^{j_{i-1}}+1+t_{i}$

for suitable $t_{i} \in\left[0,2^{j_{i}}\right)$. Thus

$$
n=2^{\mathrm{j}_{1}}+\ldots+2^{j_{i}}+2 t_{i}+1 \leq 2^{j_{1}}+\ldots+2^{j_{i}+1}-1 .
$$

Suppose $i<k$. Then $j_{i}+1 \leq j_{k}$, so $n \leq 2^{j_{1}}+\ldots+2^{j_{k}}-1=\bar{n}-2<n$ by Proposition 4(iii), a contradiction. Thus $i=k$, and

$n-2^{j_{k}}-t_{i}=2^{j_{1}}+\ldots+2^{j_{k-1}}+1+t_{i}$.

By Proposition 4(ii),

$2 t_{i}=n-\left(2^{j_{1}}+\ldots+2^{j_{k}}+1\right)=n-\bar{n}=2\left(n-2^{[\lg n]}\right)$.

Therefore $t_{i}=n-2^{[\lg n]}$. By (iii), $[\lg n]=j_{k}+1$, so

$a_{i}=n-2^{j_{k}}-t_{i}=2^{j_{k}}$.

It is straightforward to see that $2^{j_{k}}$ and $2^{j_{k}}+1$ are in $I_{L}$.

Proposition 6: The set of $b_{i}$ satisfying $b_{i}>a_{i}$ is a subinterval of $I_{L}$ except that $b_{i}=$ $n$ is not in $I_{L}$ when $n=2^{s}(s \geq 1)$. 
Proof: This is certainly true if there is no $b_{i}>a_{i}$. If there is $b_{i}>a_{i}$, then $[\lg n]=$ $j_{k}+1$ (Proposition 5(iii)). If $n \neq 2^{s}$, the two most significant 1-bits of $n_{b}$ are in positions $j_{k}+1$ and $j_{k}-q-1$, where $q$ is as defined in Proposition 5(v). Hence $n \geq$ $2^{j_{k}+1}+2^{j_{k}-q-1}$. Therefore by Proposition 4(ii), $n-\bar{n}=2\left(n-2^{j_{k}+1}\right) \geq 2^{j_{k}-q}$, or $\bar{n} \leq n-2^{j_{k}-q}$, which proves the assertion by Proposition 5(iv),(v),(vi). The exceptional case is clear.

Proposition 7: The move of B specified by $W$ always exists, that is, the vertex prescribed by $W$ for B to label, is still unlabeled prior to B's move.

Proof: The very first move of A consists of labeling some $a_{h} \in \mathscr{C}$. Then B responds by labeling $b_{h}$. This move is possible by Proposition 3. After this move, all and only all vertices between $a_{h}$ and $b_{h}$ are labeled.

Suppose, inductively, that when A labels $a_{p}$ or $b_{p}$, then B can respond by labeling $b_{p}$ or $a_{p}$ as specified by $W$, that $a_{p}$ and $b_{p}$ are on opposite sides of the interval of previously labeled vertices with $a_{p}>b_{p}$, so all and only all vertices in $\left[b_{p}, a_{p}\right]$ are now labeled.

Suppose A now labels an extreme vertex (always on the same ray), which is in $\mathscr{C}$. By Proposition 6, any extreme vertex of the form $b_{i}$ with $b_{i}>a_{i}\left(b_{i}<n\right)$ is itself of the form $a_{r}$. We may thus assume that $\mathrm{A}$ labels a vertex of the form $a_{r}=n-2^{j_{r}}$ $-t_{r}$. The response of $\mathrm{B}$ consistent with $W$ is to label $b_{r}=2^{j_{1}}+\ldots+2^{j_{r-1}}+2+$ $t_{r}$, even if $a_{r}=b_{i}$. This latter statement follows from Proposition 5(vi): the pairs $\left(a_{i}, b_{i}\right)$ with $b_{i}>a_{i}$ are symmetrically located with respect to the midpoint between the vertices $2^{j k}$ and $2^{j k}+1$. That is, for any pair $\left(a_{i}, b_{i}\right)$ with $a_{i} \in I_{L}$ and $b_{i}>a_{i}$, we have $b_{i}=a_{r}, a_{i}=b_{r}$ for suitable $r$.

Since $a_{r}$ is extreme, we have

$$
a_{r}=n-2^{j r}-t_{r}>n-2^{j p}-t_{p}=a_{p} \text {. }
$$

Thus either $r=p$ and $t_{r}<t_{p}$, or $r<p$. In either case,

$$
b_{r}=2^{j_{1}}+\ldots+2^{j_{r-1}}+2+t_{r}<2^{j_{1}}+\ldots+2^{j_{p-1}}+2+t_{p}=b_{p} \text {. }
$$

In particular, $b_{r}$ is as yet unlabeled. Moreover, $b_{r}<b_{p}<a_{p}<a_{r}$, so $b_{r}$ and $a_{r}$ are on opposite sides of the set of previously labeled vertices, and all vertices in $\left[b_{r}, a_{r}\right]$ are now labeled.

If A labeled a nonextreme vertex, that is, a vertex less than $b_{p}$, then this vertex has the form $b_{r}=2^{j_{1}}+\ldots+2^{j_{r-1}}+2+t_{r}$ for some $t_{r} \in\left[0,2^{j_{r}}\right.$ ), since the moves of the form $b_{i}$ constitute the interval $[2, \bar{n}]$ (Proposition 5(iv)). As above we then have $a_{r}>a_{p}$, where $a_{r}$ is the response of B prescribed by $W$. So again all vertices in $\left[a_{r}, b_{r}\right]$ are labeled.

Proposition 8: Every response of B as specified by $W$ is in $\mathscr{C}$ except the response of labeling $n$ when $n=2^{s}(s \geq 1)$. 
Proof: This follows from Propositions 1 and 2 if $W$ calls for labeling $b_{i}$ or $a_{i}$ with $b_{i}<a_{i}$, as a response to A's labeling of $a_{i}$ or $b_{i}$. For the case where B's response is to label $b_{i}$ with $b_{i}>a_{i}$, Proposition 6 shows that $b_{i} \in I_{L}$ (with the usual exception), hence this move is also in $\mathscr{C}$.

We now resume and complete the proof of Theorem 4. Propositions 3 and 7 show that B's response specified by $W$ is always possible. Proposition 8 shows that it is always in $\mathscr{C}$ (with the usual exception, in which $B$ wins anyway); and Proposition 1 implies that the moves in $W$ exhaust $\mathscr{C}$. Thus player A must eventually make a move in Phase I such that the next move of $B$ can transform the graph into a 0 -star, and so $\mathrm{B}$ wins.

Acknowledgment: This paper was begun while the authors met at the International Conference on Convexity and Graph Theory in Israel in 1981. It was continued by the first author while visiting the University of Calgary, Simon Fraser University and Queens College in 1984; and by the second author while visiting at Churchill College, Cambridge, Stevens Institute of Technology and Courant Institute, New York University.

\section{References}

Berlekamp E R, Conway J H and Guy R K (1982) Winning Ways, Academic Press, London Harary F, Achievement and Avoidance Games (book in preparation)

Úlehla J (1980) A complete analysis of Von Neumann's Hackendot, Int. J. Game Theory 9, 107-113

Received May 1986

Revised version August 1988 